\title{
Taller de Física Cuántica: un Método para Introducir Conceptos Fundamentales en una Actividad Extracurricular
}

\author{
Claudio A. Faúndez ${ }^{(1)}$, Yazmina G. Rojas ${ }^{(2)}$, Angela A. Pinto(3) y Hernán F. Astudillo(1) \\ (1) Depto. De Física, Facultad de Ciencias Físicas y Matemáticas, Universidad de Concepción, Barrio \\ Universitario s/n, Casilla 160-C, Concepción, Chile (e-mail: claudiofaundez@udec.cl, hastudil@udec.cl). \\ (2) Carrera de Geofísica, Facultad de Ciencias Físicas y Matemáticas, Universidad de Concepción, Barrio \\ Universitario s/n, Casilla 160-C, Concepción, Chile (e-mail: yazminarojas@udec.cl) \\ (3) Colegio Einstein, Coronel, Chile (e-mail: angelapintocampos@gmail.com)
}

Recibido Sep. 16, 2014; Aceptado Oct. 23, 2014; Versión final recibida Ene. 15, 2015

\begin{abstract}
Resumen
En este artículo se propone y analiza una metodología basada en el constructivismo y centralizada en el aprendizaje activo del estudiante. Esta metodología facilita el logro de las competencias necesarias para la adquisición de aprendizajes significativos de los fundamentos de física cuántica. El método descrito fue construido para ser desarrollado en un taller extra programático que considera los contenidos mínimos de mecánica cuántica establecidos por el Ministerio de Educación de Chile para los cursos de física. El taller fue diseñado por estudiantes universitarios de física, futuros docentes en escuelas secundarias. En el taller propuesto se entrelaza la cátedra, la actividad experimental, la discusión y la obtención de conclusiones válidas en cada actividad. Esta propuesta entrega una herramienta útil y práctica para la enseñanzaaprendizaje de la física cuántica.
\end{abstract}

Palabras clave: constructivismo, aprendizaje significativo, actividades experimentales, taller, mecánica cuántica

\section{Workshop on Quantum Physics: A Method for Introducing Fundamental Concepts in an Extracurricular Activity}

\begin{abstract}
In this paper a methodology based on constructivism and centering on active student learning, enabling students to acquire the necessary skills for achieving significant learning of the fundamentals of quantum physics is proposed and analyzed. The methodology is designed to be developed in an extra-curricular workshop program considering the minimum contents of quantum mechanics established by the Ministry of Education of Chile for the courses of physics. In the workshop, the theoretical hours, the experimental activity, the discussion and the obtaining of valid conclusions in each activity are considered. This proposal provides a useful and practical tool for the teaching-learning process of quantum physics.
\end{abstract}

Keywords: constructivism, meaningful learning, experiential activities, workshop, quantum mechanics 


\section{INTRODUCCIÓN}

La mecánica cuántica ha ejercido una fuerte influencia en nuestra sociedad, en el pensamiento humano y la filosofía (Greca y Herscovitz, 2002). Adquirimos conciencia de ello cuando observamos cotidianamente los logros, resultados y aplicaciones con que esta ciencia impacta a la sociedad mediante el desarrollo de nuevas tecnologías (Otero et al. 2009). Ejemplos relevantes para el bienestar de la sociedad van desde desarrollos como el Láser y sus implicancias médicas, la nanotecnología y sus aplicaciones a diversos procesos industriales como la industria espacial y la industria médica, entre otras. En tanto que, relevantes para el desarrollo de la ciencia podemos nombrar el Colisionador de Hadrones (LHC, por sus siglas en inglés), tecnología desarrollada para verificar interrogantes fundamentales de la ciencia (Sánchez, 2008).De aquí que la comprensión de los fundamentos de la mecánica cuántica y sus aplicaciones es de extrema relevancia para entender el medio ambiente tecnológico en que vivimos.

El logro de estos aprendizajes y sus implicancias en la formación de los alumnos, y por tanto en la sociedad, depende fuertemente de la acción docente en el aula. Siguiendo a Hodson (1993), el primer paso para asegurar que los estudiantes tengan éxito en el aprendizaje sobre la naturaleza de la mecánica cuántica, es convertir lo implícito en explícito. El segundo paso necesario es realizar una planificación conforme a un modelo científico desde un paradigma educativo caracterizado por un espacio constructivista del aprendizaje. Esto es, un modelo de ciencia que valorice la falibilidad y la dependencia teórica de la observación y del experimento, que aporte conciencia de cómo se transmiten los conocimientos dentro de la comunidad científica, y que haga hincapié en la distinción entre teorías y modelos instrumentalistas, que persiguen realizar predicciones y establecer una medida de control. Dado que la física en general tiene un carácter experimental existe consenso en el hecho de que los trabajos prácticos de laboratorio son fundamentales para su enseñanza (Barolli et al. 2010; López y Tamayo, 2012). Esto permite la conexión entre los conceptos físicos y el mundo de los sentidos, que a menudo no se observan siguiendo una instrucción tradicional (McDermott, 2013). En sistemas educacionales con recursos insuficientes es fundamental destacar la importancia que tiene para el logro de los aprendizajes de los estudiantes, la construcción de dispositivos sencillos de bajo costo que permite llevar a cabo una actividad experimental (Pérez y Falcón, 2009). En particular, como el caso de Chile, donde existen muy pocos colegios que cuentan con los recursos necesarios para realizar actividades experimentales asociadas a los contenidos mínimos obligatorios, exigidos por el Ministerio de Educación (MINEDUC) y que los docentes, en general, no cuentan con guías de trabajo experimental que permitan a los estudiantes construir su propio conocimiento a través de experimentos sencillos (Vera et al. 2013).

En un análisis exhaustivo sobre la enseñanza-aprendizaje de la mecánica cuántica en la enseñanza secundaria, Sinarcas y Solves (2013) muestran que las principales dificultades de los alumnos están en las carencias de la enseñanza que reciben a través del profesorado de Física de secundaria. Por otra parte, antecedentes de la literatura a nivel latinoamericano convienen que la falta de material para realizar actividades experimentales en esta unidad, unido a la escasez de recursos didácticos para su implementación en el aula, hace difícil abordar conceptos de mecánica cuántica a nivel de enseñanza secundaria (Bustos et al. 2013). Además, los textos que generalmente se emplean para su enseñanza no realizan sugerencias metodológicas suficientes como para favorecer significativamente las planificaciones por parte de los docentes (Ostermann y Moreira, 2000; Vicario y Venier, 2010, Sinarcas y Solves 2013). En este sentido, dentro de la formación docente universitaria en las carreras de pedagogía en ciencias, deben existir asignaturas donde los estudiantes realicen trabajos de laboratorio, en los cuales puedan tener la posibilidad de explorar, sugerir hipótesis, etc. (Gil, 1997). En este tipo de actividades experimentales es donde los estudiantes tienen que involucrarse activamente en su proceso de aprendizaje, de modo que ellos estén en todo momento intelectualmente activos (Ramírez et al. 2013).

En el presente trabajo se da a conocer una propuesta, realizada como parte de la formación universitaria de los futuros docentes de ciencias y desarrollada para la unidad mecánica cuántica. Dicha propuesta, además de generar conocimiento, potencia las habilidades de los estudiantes permitiendo despertar la curiosidad de los alumnos. La propuesta está diseñada para ser desarrollada como una actividad extracurricular enfocada a alumnos de enseñanza media chilena. Este diseño permite que alumnos desde tercero medio puedan tener acceso con antelación a lo que el programa de estudios entregado por el Ministerio de Educación decreta. Como establece Hodson (1994), podemos decir que el aprendizaje de la ciencia, de su naturaleza y de su práctica están relacionados, son necesarios y que las experiencias obtenidas con buenos resultados en cada uno de ellos, contribuye a la comprensión de los restantes, pero ninguno es suficiente por sí solo, por lo que es necesario realizar actividades prácticas con su respectiva base teórica para conocer y hacer ciencia. En línea con lo anterior, el taller propone sesiones donde cada contenido se desarrolla en tres etapas:1) Teórico- conceptual; 2) Experimental y 3) Análisis de la información y formulación de resultados. 
En las siguientes secciones damos una visión del marco teórico donde se establece la relación entre el constructivismo y las actividades experimentales y como se favorece el aprendizaje entre pares, como establece la metodología propuesta por Vigotsky (1979). Luego, describimos el diseño del taller y se muestra un análisis de los resultados y las conclusiones obtenidas.

\section{EL CONSTRUCTIVISMO Y LAS ACTIVIDADES EXPERIMENTALES}

Los tiempos de una enseñanza basada en cátedras dictadas por el profesor están quedando rezagados. Ahora, se tienen que implementar nuevas estrategias donde el profesor deje de tener una participación principal dentro del proceso de enseñanza-aprendizaje y el alumno debe ser quien ahora tome una mayor participación dentro de este proceso (García y Sánchez, 2009).

La concepción constructivista señala tres elementos básicos que determinan el estado inicial de los alumnos, al momento de iniciar un proceso de aprendizaje, estos son: i) Los alumnos presentan una determinada disposición para llevar a cabo el aprendizaje que se les plantea; ii) Esta disposición surge como resultado de la confluencia de numerosos factores de índole personal e interpersonal. Los alumnos disponen de determinadas capacidades, instrumentos, estrategia y habilidades generales para llevar a cabo el proceso de aprendizaje; y iii) Los conocimientos previos que poseen los alumnos.

La concepción constructivista considera indispensable el tercer aspecto antes de comenzar un proceso de aprendizaje. Según lo que propone Ausubel et al. (1983), el aprendizaje significativo depende tanto de las capacidades y motivaciones del estudiante como de sus conocimientos previos. ¿Cómo es posible contactar en un primer momento con el nuevo conocimiento?. Pues, a partir de algo que ya conocemos y sabemos. Así, gracias a lo que el alumno ya sabe, puede hacer una primera lectura del nuevo contenido, atribuirle un primer nivel de significado y sentido e iniciar el proceso de aprendizaje del mismo. Tal como afirma Moreira (2008), las nuevas ideas, conceptos y proposiciones pueden ser aprendidos significativamente en la medida en que otras ideas, conceptos, proposiciones, relevantes e inclusivos, estén adecuadamente claros y disponibles en la estructura cognitiva del individuo y funcionen como punto de anclaje de los primeros.

Una estrategia para la enseñanza de la mecánica cuántica que responde a lo anterior son las actividades experimentales, dado su carácter de ciencia experimental existe consenso en el hecho de que los trabajos prácticos de laboratorio son fundamentales para su enseñanza. Su importancia radica no sólo en la posibilidad de observación y experimentación sobre la realidad y de desarrollo de habilidades experimentales, sino también, y quizás más fuertemente, en la posibilidad que brindan para relacionar las teorías y modelos con la experiencia, y suministrar así, una oportunidad para que los alumnos conozcan cómo se construye el conocimiento científico (Hodson, 1994; Jaime y Escudero, 2011). Estas experiencias permiten que se produzca en ellos un aprendizaje significativo y autónomo. Afirmando lo anterior, Álvarez et al. (2008) y Flores et al. (2009) señalan que las experiencias son la clave para la comprensión conceptual por parte de los estudiantes y para la construcción del conocimiento del mundo que los rodea.

Actualmente, Seré (2002) propone algunas ventajas de las actividades experimentales las cuales se nombran en la tabla 1. Como se puede observar de las características listadas, los verbos se encuentran en diferentes niveles cognitivos, por ejemplo, si nos basamos en la taxonomía de Bloom (Bloom, 1976) encontramos desde el nivel de conocimiento hasta el nivel de análisis.

Tabla 1: Ventajas de las actividades experimentales propuesto por Serè (2002)

\begin{tabular}{|l|l|}
\hline Comprender la teoría & Aprender a usar el saber teórico aprendido \\
Aprender la teoría & Motivación \\
Realizar experiencias & Interés de razonar \\
Aprender a rehacer & Interés de visualizar los objetos y eventos \\
Aprender los procedimientos & \\
\hline
\end{tabular}

Uno de los puntos relevantes del paradigma constructivista se basa en generar aprendizaje significativo en los alumnos propuesto por Ausubel (1983) y esto se logra anclando los conocimiento previos con el nuevo conocimiento. Además, algunos filósofos han expresado que el pensamiento es más completo cuando es capaz de poner en juicio y tomar en cuenta la dimensión de la acción. Así, Bergson pregona «pensar como un hombre de acción, actuar como un hombre de pensamiento».También Vigotsky (1979) señala que el aprendizaje se logra entre pares, es decir, resulta fundamentalmente de la colaboración y cooperación, por lo tanto, el trabajo experimental es una forma de potenciar el aprendizaje entre alumnos. 
El taller es un método de organizar el proceso de enseñanza- aprendizaje integrando reflexivamente teoría y práctica, para abordar la resolución de un problema o el estudio de algún contenido, mediante la actividad participativa del alumno, el ensayo creativo de sus capacidades, conocimientos y destrezas y utilizando múltiples recursos y materiales (Maya, 2007).

\section{DESCRIPCIÓN DEL TALLER}

Estudiantes universitarios de física de la Universidad de Concepción, futuros docentes de escuela secundaria, desarrollaron un taller con fines de enseñanza. Específicamente, el taller consiste en 14 sesiones de 2 horas pedagógicas (90 minutos). En el transcurso del taller se tratan los temas de mecánica cuántica que se encuentran en el programa de estudio de cuarto año medio, formación diferenciada (MINEDUC, 2002), así como otros temas de física cuántica relevantes para la comprensión de la ciencia y tecnología. Estos se encuentran resumidos en la tabla 2:

Cada experiencia tiene las siguientes fases: teórico-conceptual, experimental y de análisis de la información y formulación de resultados:

1. Teórico- conceptual: Se revisan y sustentan los conocimientos previos necesarios para la enseñanza del nuevo concepto siendo el profesor un guía para el alumno en el proceso.

2. Experimental: Mediante guías de trabajo se induce al alumno a construir, montar y realizar los experimentos establecidos. En esta fase, los alumnos generan su propio ambiente de aprendizaje, construyen y modifican los dispositivos para observar los fenómenos y formulan hipótesis.

3. Análisis de la información y formulación de resultados: Luego de observar y relacionar lo experimentado con la teoría, los alumnos analizan, describen, explican, discuten los conceptos involucrados en el fenómeno con sus pares y formulan las conclusiones y análisis correspondientes favoreciendo así el aprendizaje de tipo colaborativo.

Tabla 2: Contenidos del taller de mecánica cuántica

\begin{tabular}{|c|c|}
\hline & Unidad 3: El mundo cuántico \\
\hline Contenidos mínimos & $\begin{array}{l}\text { - Dualidad onda corpúsculo en la materia. Relaciones de Louis De Broglie. } \\
\text { - Análisis del átomo de hidrógeno de Niels Bohr a la luz de las relaciones de De Broglie. } \\
\text { - Noción de función de onda y sus consecuencias sobre la descripción del átomo. } \\
\text { - Interpretaciones acerca de sus significados. }\end{array}$ \\
\hline Contenidos extras & $\begin{array}{l}\text { - Superconductor. } \\
\text { - Laser. } \\
\text { - Imagen por resonancia magnética nuclear (MRI). } \\
\text { - Física estadística (Fermi-Dirac, Maxwell-Boltzmann y Bose-Einstein). }\end{array}$ \\
\hline
\end{tabular}

El taller contiene 6 actividades experimentales de gran contenido conceptual como se detalla en la Tabla 3 . Cada actividad experimental tiene sus respectivas guías de aprendizaje que incluyen: los materiales de la actividad, el montaje experimental o construcción del instrumento, preguntas que guían al análisis de la experiencia y motivan al intercambio de opiniones entre pares. Todos los contenidos se introducen mediante la utilización de TICs (Urréjola et al. 2011; Faúndez et al. 2014) y la detección de los conocimientos previos del alumno se realizan mediante preguntas por el profesor y la aplicación de test de entrada. De esta forma se crean las condiciones para generar un ambiente de aprendizaje caracterizado por el trabajo colectivo, enfatizando el análisis e interpretación que los propios alumnos hacen de los temas propuestos. En particular, para las actividades experimentales: electroscopio casero, la doble rendija de Young y espectroscopio casero, los dispositivos experimentales para la observación de los fenómenos son construidos de forma individual por los alumnos.

Tabla 3: Contenidos y actividades experimentales abordadas en el taller.

\begin{tabular}{|l|l|c|}
\hline Contenido & Actividad experimental & Número de horas \\
\hline Efecto fotoeléctrico & Electroscopio casero & 2 horas \\
\hline Efecto fotoeléctrico & Constante de Planck & 2 horas \\
\hline Difracción e interferencia & Experimento de la doble rendija de Young & 4 horas \\
\hline Espectros atómicos & Espectroscopio casero (Heredia , 2009) & 4 horas \\
\hline Física estadística & Estadística de Maxwell- Boltzman & 2 horas \\
\hline Superconductividad & Efecto Meissner & 2 horas \\
\hline
\end{tabular}


Tabla 4: Cuadro resumen de los experimentos que contiene el taller.

\begin{tabular}{|c|c|c|c|}
\hline Tema & Laboratorio & Objetivos & Materiales \\
\hline Efecto fotoeléctrico & $\begin{array}{l}\text { Constante de } \\
\text { Planck }\end{array}$ & $\begin{array}{l}\text { Conocer y comprender en qué } \\
\text { consiste el efecto fotoeléctrico. } \\
\text { Calcular experimentalmente la } \\
\text { constante de Planck. }\end{array}$ & $\begin{array}{l}\text { - } 1 \text { potenciómetro } \\
\text { - } 5 \text { LED de diferentes Colores. } \\
\text { - Cable. } \\
\text { - } 1 \text { multitester. } \\
\text { - } 1 \text { batería de } 9 \mathrm{~V}\end{array}$ \\
\hline Efecto fotoeléctrico & $\begin{array}{l}\text { Espectroscopio } \\
\text { Casero }\end{array}$ & $\begin{array}{l}\text { Analizar el efecto fotoeléctrico } \\
\text { con las descargas eléctricas } \\
\text { producidas con la televisión. }\end{array}$ & $\begin{array}{l}\text { - } 1 \text { frasco de vidrio. } \\
\text { - Papel aluminio. } \\
\text {-1corcho. } \\
\text { - Alambre conductor. }\end{array}$ \\
\hline $\begin{array}{l}\text { Difracción e } \\
\text { interferencia }\end{array}$ & $\begin{array}{l}\text { Experimento de } \\
\text { la doble rendija } \\
\text { de Young }\end{array}$ & $\begin{array}{l}\text { Observar el patrón formado por } \\
\text { la luz al pasar por la doble } \\
\text { rendija. }\end{array}$ & $\begin{array}{l}\text { - Papel aluminio. } \\
\text { - Alfiler. } \\
\text { - Vidrio porta objetos. } \\
\text { - } 1 \text { láser. } \\
\text { - Una doble ranura. } \\
\text { - Una hoja de papel blanco. }\end{array}$ \\
\hline $\begin{array}{l}\text { Espectros } \\
\text { atómicos }\end{array}$ & $\begin{array}{l}\text { Espectroscopio } \\
\text { Casero }\end{array}$ & $\begin{array}{l}\text { Observar y analizar la luz al } \\
\text { contacto con una rendija de } \\
\text { difracción. }\end{array}$ & $\begin{array}{l}\text { - } 1 \text { CD. } \\
\text { - Cartón forrado. } \\
\text { - Cartón de un rollo de papel higiénico. } \\
\text { - Papel aluminio. } \\
\text { - Pegamento. } \\
\text { - Cinta aislante negra. } \\
\text { - Cinta scotch. } \\
\text { - Tijeras. } \\
\text { - Fuentes luminosas diversas. } \\
\text { - Filtros de colores. }\end{array}$ \\
\hline Física estadística & $\begin{array}{l}\text { Estadística de } \\
\text { Maxwell y } \\
\text { Boltzmann }\end{array}$ & $\begin{array}{l}\text { Conocer y comprender las } \\
\text { estadísticas de Maxwell- } \\
\text { Boltzmann, Fermi- Dirac y } \\
\text { Bose- Einstein. }\end{array}$ & $\begin{array}{l}\text { - } 1 \text { termistor. } \\
\text { - } 1 \text { termómetro. } \\
\text { - } 1 \text { Recipiente. } \\
\text { - } 1 \text { mechero. } \\
\text { - } 1 \text { Batería. } \\
\text { - } 1 \text { amperímetro. } \\
\text { - cables. } \\
\text { - } 1 \text { calorímetro. }\end{array}$ \\
\hline Superconductividad & Efecto Meissner & $\begin{array}{l}\text { Observar y analizar el efecto } \\
\text { Meissner. }\end{array}$ & $\begin{array}{l}\text { - Kit superconductor (recipiente, imán, } \\
\text { superconductor, pinzas, antiparras). } \\
\text { - Nitrógeno líquido. }\end{array}$ \\
\hline
\end{tabular}

En la tabla 4, se describen en forma general los experimentos propuestos en el taller. Una descripción detallada de cada uno de ellos se encuentra disponible como material suplementario.

\section{EXPERIMENTO DEL EFECTO FOTOELÉCTRICO : "CONSTANTE DE PLANCK”.}

De los experimentos señalados en la Tabla 4, a modo de ejemplo, a continuación se describe del tema efecto fotoeléctrico el laboratorio del cálculo de la Constante de Planck. Los análisis y resultados obtenidos por los estudiantes son presentados al profesor. Por otro lado, se incluyen las respuestas y observaciones esperadas en el desarrollo de la actividad experimental y la posterior orientación al profesor para motivar la discusión.

\section{Protocolo de medición (ver guía experimental)}

1- Registre el voltaje que indica el multitester cuando se obtiene el primer rayo de luz.

2- Anote la longitud de onda a la cual corresponde la luz del LED.

3- Realice los pasos anteriores para cada uno de los LED de color.

\section{Protocolo de Resultados}

4- Considere el valor de la velocidad de la luz (c) y el valor de la carga de un electrón (e).

5- Considere la relación de Einstein para el efecto fotoeléctrico y utilice los datos obtenidos.

6- Calcule el valor de la constante de Planck (h) para cada color.

7- Obtenga el valor promedio de $\mathrm{h}$. 


\section{Protocolo de análisis}

8- Explique las relaciones que existen entre las variables de la Ecuación de Einstein.

9- De acuerdo a las constantes obtenidas para cada color ¿son similares al valor aceptado de la constante de Planck? Fundamente su respuesta.

10- Indique posibles factores que influyen en la diferencia del valor aceptado de $\mathrm{h}$ y el experimental obtenido por usted.

11- Redacte conclusiones del experimento.

\section{RESULTADOS}

Los resultados obtenidos de la experiencia se muestran en la Tabla 5. Se muestra el voltaje, la longitud de onda, la velocidad de la luz, la frecuencia, la carga del electrón y la constante de Planck calculada. Estos resultados se obtuvieron de la siguiente forma:

i) En la tabla se anotará primero la longitud de onda para cada color de luz que emiten los LED (dadas por el profesor).

ii) Luego, armado el circuito medirán el voltaje (mínimo voltaje que requiere el LED para expulsar los electrones) cuando el LED expulse el primer haz de luz. Para cada LED los voltajes deben ser distintos y estos aumentan a medida que disminuye la longitud de onda.

iii) EI LED posee una estructura en el cual podemos decir que ocurre el efecto fotoeléctrico en su interior, ya que posee una lámina metálica y a través de un circuito se le entrega suficiente energía para que se emita un fotón. Por lo tanto, se cumple el efecto fotoeléctrico, expresando la energía cinética máxima como se muestra en la ecn. (1).

Tabla 5: Resultados obtenidos de la experiencia.

\begin{tabular}{|l|c|c|c|c|c|c|}
\hline & $\begin{array}{c}\text { Voltaje } \\
(\mathrm{V})\end{array}$ & $\begin{array}{c}\text { Longitud de } \\
\text { onda }(\mathrm{m})\end{array}$ & $\begin{array}{c}\text { Velocidad } \\
\text { de la luz }(\mathrm{m} / \mathrm{s})\end{array}$ & $\begin{array}{c}\text { Frecuencia } \\
(\mathrm{Hz})\end{array}$ & $\begin{array}{c}\text { Carga del } \\
\text { electrón(C) }\end{array}$ & $\begin{array}{c}\text { Constante de } \\
\text { Planck(Js) }\end{array}$ \\
\hline $1^{\circ}$ LED & 1,44 & $\begin{array}{c}750 \times 10^{-9} \\
\text { (rojo) }\end{array}$ & $3 \times 10^{8}$ & $4,00 \times 10^{14}$ & $1,6 \times 10^{-19}$ & $5,76 \times 10^{-34}$ \\
\hline $2^{\circ}$ LED & 1,56 & $\begin{array}{c}590 \times 10^{-9} \\
\text { (amarillo) }\end{array}$ & $3 \times 10^{8}$ & $5,08 \times 10^{14}$ & $1,6 \times 10^{-19}$ & $4,91 \times 10^{-34}$ \\
\hline $3^{\circ}$ LED & 1,90 & $\begin{array}{c}570 \times 10^{-9} \\
\text { (verde) }\end{array}$ & $3 \times 10^{8}$ & $5,26 \times 10^{14}$ & $1,6 \times 10^{-19}$ & $5,78 \times 10^{-34}$ \\
\hline $4^{\circ}$ LED & 2,12 & $\begin{array}{c}495 \times 10^{-9} \\
(\text { azul })\end{array}$ & $3 \times 10^{8}$ & $6.06 \times 10^{14}$ & $1,6 \times 10^{-19}$ & $5,60 \times 10^{-34}$ \\
\hline $5^{\circ}$ LED & 2,48 & $\begin{array}{c}450 \times 10^{-9} \\
(\text { violeta })\end{array}$ & $3 \times 10^{8}$ & $6,67 \times 10^{14}$ & $1,6 \times 10^{-19}$ & $5,95 \times 10^{-34}$ \\
\hline
\end{tabular}

$K_{\max }=e V_{S} \quad$ y $K_{\max }=h f-\phi$

En esta ecuación,

$K_{\max }$ : Energía cinética máxima de los fotoelectrones.

e: carga del electrón $1.6 \times 10^{-19} \mathrm{C}$.

$V_{s}$ : Potencial de frenado.

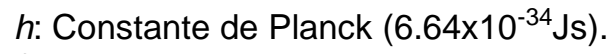

$f$ : Frecuencia de corte.

$\phi$ : Función de trabajo del metal, energía mínima con la cual el electrón está ligado al metal.

Para el caso de los LED: $\phi=0$, debido a que no existe una energía mínima para la expulsión del electrón cuando en el LED circula corriente.

Luego igualando se obtiene: 
$e V=h f$

Despejando $h$, se obtiene:

$h=\frac{e V}{f}$

Por lo tanto, la variable que falta encontrar para calcular la constante de Planck es la frecuencia de los colores de cada LED.

Se debe recordar que tenemos la longitud de onda para cada color y de esta podemos calcular la frecuencia a través de:

$f=\frac{c}{\lambda}$

En esta ecuación, c corresponde a la velocidad de la luz $\left(3 \times 10^{8} \mathrm{~m} / \mathrm{s}\right)$ y $\lambda$ es la longitud de onda.

Ahora se cuenta con toda la información necesaria para calcular la constante de Planck para cada color, luego se obtiene un valor promedio de todas las constantes de Planck obtenidas del experimento $(<h>=$ $\left.5,60 \times 10^{-34} \mathrm{~J} \cdot \mathrm{s}\right)$. El valor aceptado de la constante de Planck es $6,62 \times 10^{-34} \mathrm{~J} \cdot \mathrm{s}$. Esto indica que el valor obtenido en el experimento es un $15.4 \%$ menor que el aceptado.

Ejemplo, para el LED que emite luz violeta.

Voltaje: 2,48 V (Medida por los alumnos a través de un Tester).

Longitud de onda: $450 \times 10^{-9} \mathrm{~m}$ (Buscado por los alumnos en la web).

Velocidad de la luz: $3 \times 10^{8} \mathrm{~m} / \mathrm{s}$

Frecuencia:

$f=\frac{c}{\lambda}=\frac{3 \times 10^{8}\left(\frac{\mathrm{m}}{\mathrm{s}}\right)}{450 \times 10^{-9}(\mathrm{~m})}=6,67 \times 10^{14}(\mathrm{~Hz})$

Carga del electrón: $1,6 \times 10^{-19} \mathrm{c}$

Constante de Planck:

$h=\frac{e V}{f}=\frac{\left(1,6 \times 10^{-19} \mathrm{c}\right)(2,48 \mathrm{v})}{6,67 \times 10^{14} \mathrm{~Hz}}=5,95 \times 10^{-34}(\mathrm{~J} \cdot \mathrm{s})$

\section{Implementación de la Propuesta}

La propuesta de este trabajo ha sido implementada realizando una de las experiencias, correspondiente al laboratorio del espectroscopio casero y análisis de espectros atómicos (ver tabla 4). La experiencia se realizó en un Colegio de la octava región de Chile y el grupo de trabajo conto con la participación de 15 alumnos entre los 16 y 17 años de edad. Antes del trabajo de laboratorio, se realizó un pre test sobre el tema de espectros atómicos y al finalizar la experiencia se realizó un pos test obteniendo un factor de Hake equivalente a 0.87 , es decir, una alta ganancia conceptual del aprendizaje adquirido.

Los alumnos durante el proceso realizaron un informe donde debían incluir los siguientes aspectos: objetivos del experimento, introducción, metodología, análisis, comentarios y conclusiones. Durante el proceso y finalización de la actividad, se logró apreciar las siguientes competencias adquiridas entre los alumnos: trabajar en equipo, describir los distintos tipos de espectros atómicos, discutir las ideas con sus pares, asociar el concepto de espectro atómico con el laboratorio, justificar sus ideas, identificar qué tipo de espectro corresponde a diversas fuentes de luz y rescatar ideas fundamentales del laboratorio. 


\section{CONCLUSIONES}

Estudiantes universitarios de física de la Universidad de Concepción, propusieron un método para ser desarrollado en un taller extra programático, que incluye contenidos mínimos de mecánica cuántica establecidos por el MINEDUC de Chile, los cuales corresponden al efecto fotoeléctrico, física estadística, superconductividad, entre otros, que han sido especificadas en el trabajo.

La propuesta responde claramente al interrogante ¿Cómo generar aprendizaje significativo sobre los contenidos de mecánica cuántica en los alumnos de enseñanza media?. Más aún, facilita herramientas que permiten una mayor comprensión de los temas fundamentales de la mecánica cuántica: por medio de experiencias prácticas donde el alumno puede ver, construir, interactuar y concluir, logrando los aprendizajes mínimos requeridos. De lo anterior, podemos extraer las siguientes conclusiones.

i) El estudio está basado en realizar experimentos fundamentales de la mecánica cuántica, que pueden ser llevados al aula sin mayor dificultad, ya que son diseñados para ser construidos con materiales de fácil acceso.

ii) Esta estrategia permite que los alumnos se interioricen en los fundamentos de la mecánica cuántica generando aprendizajes significativos mediante el desarrollo de actividades experimentales.

iii) Además permite que los alumnos desarrollen sus habilidades para que se comporten como un receptor activo, en el cual, el profesor solo es el guía de dicho conocimiento ayudando al alumno a llegar a la respuesta correcta. En esta etapa se logra que los alumnos aprendan entre pares, respetando la opinión de los demás y los diferentes puntos de vista de las actividades, eliminando y refutando ideas.

iv) Logra abordar los aprendizajes esperados mínimos sugeridos por el MINEDUC, permitiendo que el alumno aumente su aprendizaje cognitivo, desde lo más simple (conocer) hasta lo más complejo (analizar) de forma jerárquica.

\section{MATERIAL SUPLEMENTARIO}

Se dispone de material adicional que incluye una descripción detallada de los seis laboratorios propuestos en este trabajo. Se puede solicitar el material al Dr. Claudio A. Faúndez (claudiofaundez@udec.cl).

\section{AGRADECIMIENTOS}

Los autores agradecen al Departamento de Física y la Dirección de Docencia de la Universidad de Concepción por el apoyo en la construcción de este trabajo, y a los miembros del taller de Micro Didáctica Física del Departamento de Física por su colaboración.

\section{REFERENCIAS}

Alvarez, F., Rodríguez-Pérez, J.R., Sanz-Ablanedo, E., Fernández-Martínez, M. Aprender enseñando: Elaboración de materiales didácticos que facilitan el aprendizaje autónomo. Formación Universitaria, 1 (6), 19-28, (2008).

Ausubel, D., Novak, J y Hanesian, H. Psicología educativa: un punto de vista cognoscitivo. México:Trillas(1983).

Barolli, E., Laburú, C.E., Guridi, V.M. Laboratorio didáctico de ciencias: Caminos de investigación. Revista Electrónica de Enseñanza de las Ciencias, 9(1), 88-110, (2010).

Bloom, B. Taxonomía de los objetivos de la educación (Tercera edición). Alcoy: Marfil(1979).

Bustos, L., Otaíza, A., Yupangui, M. La física Moderna en la educación media. Eureka, Enseñanza de las ciencias físicas, Memorias y Tesinas, 29-31, Agosto (2013).

Faúndez, C.A., Bravo, A.A., Melo, A.D., Astudillo, H.F. Laboratorio virtual para la unidad tierra y universo como parte de la formación universitaria de docentes de ciencias. Formación Universitaria, 7(3), 33-40, (2014). 
Flores, J., Caballero, M.C., Moreira, M.A. El laboratorio en la enseñanza de las ciencias: Una visión integral en este complejo ambiente de aprendizaje. Revista de Investigación, 68 (33), 75-111, (2009).

García, R. y Sánchez, D. La enseñanza de conceptos físicos en secundaria: diseño de secuencias didácticas que incorporan diversos tipos de actividades. Lat. Am. J. Phys. Educ. 3(1), 62-67, (2009).

Gil, S. Nuevas tecnologías en la enseñanza de la física oportunidades y desafíos. Memoria VI Conferencia Interamericana sobre educación en la Física (1997).

Greca, I.M. y Herscovitz, V.E. Construyendo significado en mecánica cuántica: fundamentación y resultados de una propuesta innovadora para su introducción en el nivel universitario. Enseñanza de las ciencias, 20(2), 327-338, (2002).

Hodson, D. Teaching and learning about science: considerations in the philosophy and sociology of science, en Edwards, D. y Scanlon, E. (eds.), Issues in Science Education: Teaching, Learning and Assessment(1993).

Hodson, D. Hacia un enfoque más crítico del trabajo de laboratorio. Enseñanza de las ciencias, 12(3), 299313,(1994).

Heredia, S. Como construir un espectroscopio casero con un CD. Revista Eureka sobre Enseñanza y Divulgación de las Ciencias. 6(3), 491-495, (2009).

Jaime, E y Escudero, C. El trabajo experimental como posible generador de conocimiento en enseñanza de la física. Enseñanza de las ciencias. 29(3), 371-380, (2011).

López, A.M., y Tamayo, O.E. Las prácticas de laboratorio en la enseñanza de las ciencias naturales. Latinoam.estud. educ. Manizales (Colombia), 8(1), 145-166 (2012).

Maya, A. El Taller educativo. ¿Qué es? Fundamentos, cómo organizarlo y dirigirlo, cómo evaluarlo. Colección Aula Abierta. Libro ISBN: 978-958-20-0277-8. Bogotá, D.C. Colombia (2007).

McDermott, L.C. Improving the teaching of science through discipline-based education research: An example from physics. European Journal of Science and Mathematics Education, 1(1), 1-12, (2013).

MINEDUC. Física, Programa de estudios cuarto año medio formación diferenciada (Tercera edición). Chile(2002).

Moreira, M. Organizadores previos y aprendizaje significativo. Revista Chilena de Educación científica, 7(2), 23-30, (2008).

Pérez, E., Falcón, N. Diseño de prototipos experimentales orientados al aprendizaje de la óptica. Revista Eureka sobre Enseñanza y Divulgación de las Ciencias, 6(3), 452-465, (2009).

Otero, M.R., Fanaro, M.A., Arlego, M. Investigación y desarrollo de propuestas didácticas para la enseñanza de la física en la escuela secundaria: nociones cuánticas. Revista electrónica de investigación en educación en ciencias, año 4(1), 59-74, (2009).

Ostermann, F. y Moreira, M. Investigación didáctica: física contemporánea en la escuela secundaria: Una experiencia en el aula involucrando formación de profesores. Enseñanza de las ciencias, 18(3), 391-404, (2000).

Ramírez, G.Y., López, A., Ramírez, M. Clases demostrativas interactivas de magnetismo en el bachillerato del IPN. Lat. Am. J. Phys. Educ., 7(1), 27-36, (2013).

Sánchez, G. El gran acelerador de hadrones (LHC) y la búsqueda de la partícula divina. NUCLEAR ESPAÑA octubre, ISSN/ISBN: 1137-2885, (2008).

Sinarcas, V. y Solves, J. Dificultades en el aprendizaje y la enseñanza de la física cuántica en el bachillerato. Enseñanza de las Ciencias. Revista de Investigación y Experiencias Didácticas,31 (3), 9-25, (2013). 
Séré, M. La enseñanza en el laboratorio. ¿Qué podemos aprender en términos de conocimiento práctico y de actitudes hacia la ciencia?. Enseñanza de las ciencias, 20(3), 357-368 (2002).

Urréjola, S., Valderrama, J.O., Sánchez, A., Aplicación de las nuevas tecnologías a la colaboración docente entre universidades de distintos continentes. Proyecto AECID entre la Universidad de La Serena (Chile) y la Universidad de Vigo (España). Editorial: Nova Galicia Edicíons, Vigo-España (2011).

Vera, F., Rivera, Fuentes. R., La galería de Galileo: videos de experimentos para la enseñanza de la física. Revista Estudio Pedagógicos, 39, 143-151 (2013).

Vicario, J.E. yVenier, F.L. La enseñanza de la física moderna, en debate en Latinoamérica. Revista Argentina de Enseñanza de la Ingeniería, Año 11(20), 49-57 (2010).

Vigotsky, L. Pensamiento y Lenguaje. La Pléyade. Buenos Aires (1979). 